\title{
Optimization and Development of ITO-Free Plasmonic Gold Nanoparticles Assisted Inverted Organic Solar Cells
}

\section{Vishal Gupta}

University of Delhi

\section{Anju Srivastava}

Hindu College, University of Delhi

\section{Reena Jain}

Hindu College, University of Delhi

\section{Vijay Kumar Sharma}

Shyam Lal College, University of Delhi

Lalit Kumar ( $\nabla$ lalitkrchauhan@gmail.com )

Hindu College, University of Delhi

\section{Research Article}

Keywords: Inverted organic solar cell, Plasmonic nanoparticles, UV filtering layer, PCE and stability enhancement

Posted Date: January 14th, 2022

DOI: https://doi.org/10.21203/rs.3.rs-1249295/v1

License: (c) (i) This work is licensed under a Creative Commons Attribution 4.0 International License. Read Full License 


\section{Abstract}

This paper focuses on the fabrication of an ITO-free plasmonic assisted inverted organic solar cell (OSC) constituting aluminium doped zinc oxide (AZO) as front cathode and ultraviolet (UV) filtering layer. The gold nanoflowers are introduced in the device to increase the efficiency using localized surface plasmon resonance (LSPR) shown by plasmonic nanoparticles. We used GPVDM software to first optimize the cell, based on the geometry AZO/ZnO/PTB7:PC71BM/MoO3/Ag where AZO acts as the transparent conducting oxide (TCO) cathode and UV filter, zinc oxide ( $\mathrm{ZnO})$ behaves as the electron transport layer (ETL), Thieno[3,4 b]thiophene-alt-benzodithiophene: [6,6]-phenyl C71 butyric acid methyl ester (PTB7: $\mathrm{PC71BM}$ ) mixture as the active layer, molybdenum trioxide (MoO3) as the hole transport layer ( $\mathrm{HTL}$ ) and silver ( $\mathrm{Ag}$ ) serves as the anode layer. By modelling, we find that the optimized device with maximum power conversion efficiency (PCE) includes $10 \mathrm{~nm}$ thick HTL, $200 \mathrm{~nm}$ thick photoactive layer and ETL thickness of $30 \mathrm{~nm}$. Using the optimized thicknesses, we have fabricated three structurally identical inverted OSCs: first having AZO as the front cathode (AZO based device); second with ITO as the front cathode (ITO based control device); third includes AZO as cathode and plasmonic gold nanoflowers embedded inside the active layer (plasmonic assisted AZO based device). The AZO based device exhibited the PCE value of $6.19 \%$, slightly less than the efficiency of $6.83 \%$ for ITO based control device. However, a remarkable increase in the lifetime was achieved for AZO based device under UV assisted acceleration ageing test. The stability enhancement of AZO based device is because of the UV filtering properties of AZO which prevent degradation in the device due to UV exposure. Also, the PCE of AZO based device was further enhanced to $7.01 \%$ when plasmonic gold nanoparticles were included in the active layer. This work provides a feasible way to develop an ITO free plasmonic assisted inverted organic solar cell to achieve cost-effectiveness, high efficiency and stability.

\section{Introduction}

Sun is the most powerful and sustainable energy source available on the planet. Even though the earth receives only a small part of the sun's enormous energy, an hour of solar energy is equivalent to the energy consumed by humans in an entire year[1]. Photovoltaic (PV) devices exhibit direct and efficient solar energy to electrical energy conversion. Organic solar cells (OSCs) have enormous potential in solar energy conversion technology because of interesting associated properties like economical large scale production, greater mechanical flexibility, dynamic structural variation etc[2, 3]. Also, OSC modules are thin, lightweight and flexible that can be installed easily on any regular structure like buildings or irregularly shaped structures like tents or even fabrics[4,5]. Though the field of OSCs is in substantial progress, there are hurdles to boost its commercial realization, like low power conversion efficiency, less lifetime and inability for large scale production[6]. One of the prominent problems is the exploitation of indium tin oxide (ITO) as a cathode material. Due to the scarcity of indium, a multifold price rise is there for indium which has implicitly led to the increased cost of ITO based OSCs[7, 8]. AZO is a potential substitute for ITO because of its concomitant properties like high electrical conductivity, good optical transmission and economical cost $[9,10]$. In addition, AZO has a bandgap around $3.26 \mathrm{eV}$ due to which it 
can efficiently block UV rays and restrict the device from degradation. There are reports on developing OSCs with inverted structures, having efficiencies comparable to regular structure OSCs, with a lot of scope for stability improvement and the ability to result in roll-to-roll mass production[11, 12].

Nowadays, bulk heterojunction (BHJ) OSCs utilizing polymer-fullerene blend show great potential in photovoltaic devices by virtue of their solution-processable and efficient exciton dissociation properties. There have been tremendous efforts by researchers all over the world to enhance the PCE of the OSCs by employing various strategies like using novel active and interfacial materials, varying device architecture, introducing plasmonic nanoparticles, using more efficient device fabrication techniques etc.[13]. The PCE mainly depends upon the absorption of photons inside the photoactive layer. To increase the photon absorption, the active layer thickness should be high. However, a highly thick active layer results in increased device resistance and more exciton recombination because of the low mobility of carriers and low diffusion length of exciton inside the active layer. Thus, it is imperative to use some other lighttrapping techniques to enhance photon absorption inside the active layer while keeping the optimum thickness. There are reports on the introduction of noble metallic nanoparticles into organic photovoltaic devices to enhance light absorption through LSPR shown by plasmonic nanoparticles[14]. Here we report a 13\% enhancement in PCE by adding gold nanoflowers inside the photoactive layer of the AZO based inverted OSC. The incorporation of gold nanoflowers exhibit the advantages of light absorption enhancement over a wide range, increased generation rate of exciton, higher exciton dissociation efficiency and charge carrier density enhancement which result in higher performance of the device[1517].

We first theoretically investigated the effect of variation in ETL, active layer and HTL thickness on PCE and other critical parameters of OSC and optimized the same[18]. Then subsequently, three devices with the optimized thickness (AZO based device, ITO based control device, plasmonic assisted AZO based device) were fabricated and parameters like $V_{O C}, J_{S C}, P_{\text {max }}, F F$ and PCE were tabulated from the current density-voltage $(\mathrm{J}-\mathrm{V})$ measurements. The goal of the present study is to fabricate cost-effective, highly efficient and stable ITO free plasmonic assisted inverted OSC by replacing expensive ITO with economical aluminium doped zinc oxide (AZO) and introducing gold nanoflowers inside the active layer. The bilayer geometry consisting of $\mathrm{ZnO}$ and $\mathrm{AZO}$ is utilized for better extraction of electrons[19-21]. Fabrication of AZO based plasmonic OSC is important for economical viability, better performance and stability enhancement, as it will drastically trim the cost of production, increase efficiency and enhance the lifetime of the device[22].

\section{Methods}

\section{Device Modelling}

The ITO free inverted OSC geometry is depicted in Fig. 1(a). The OSC consists of five layers viz:- AZO (transparent bottom cathode), $\mathrm{ZnO}(\mathrm{ETL}), \mathrm{PTB} 7: \mathrm{PC}_{71} \mathrm{BM}$ blend (active layer), $\mathrm{MoO}_{3}(\mathrm{HTL}), \mathrm{Ag}$ (top anode) $[23,24]$. The energy levels of the different layers of the OSC are depicted in Fig. 1(b). All the simulations 
are done using General Purpose Photovoltaic Device Model (GPVDM) solar simulator software[25]. We have investigated the electrical properties of the device through the GPVDM simulations. GPVDM solves poisson's, drift-diffusion and continuity equations for both electrons and holes. The drift-diffusion equations are solved in coordinate space using the finite difference method to explain the flow of charge inside the device[26]. This simulation software solves the carrier trapping and escape equations in energy space corresponding to each mesh point in coordinate space. Therefore, GPVDM resolves the carrier density in both position and energy space. Shockley-Read-Hall (SRH) recombination is utilized to explain the recombination of the carrier in the 1D and time domain[27].

The drift-diffusion equations (electrons and holes) are solved in position space to explain charge carrier transport.

$$
\begin{aligned}
& J_{n}=q \mu_{e} n_{f} \frac{\partial E_{\text {LUMO }}}{\partial x}+q D_{n} \frac{\partial n_{f}}{\partial x} \\
& J_{p}=q \mu_{h} p_{f} \frac{\partial E_{\text {HOMO }}}{\partial x}-q D_{p} \frac{\partial p_{f}}{\partial x}
\end{aligned}
$$

The continuity equations (electrons and holes) are used to preserve the conservation of charge carriers.

$$
\begin{aligned}
& \frac{\partial I_{n}}{\partial x}=q(R-G) \\
& \frac{\partial J_{p}}{\partial x}=-q(R-G)
\end{aligned}
$$

where $\mathrm{R}=$ net recombination rate per unit volume and $\mathrm{G}=$ net generation rate per unit volume

The internal potential distribution inside the cell is calculated using poisson's equation.

$$
\frac{d}{d x} \epsilon_{0} \epsilon_{r} \frac{d \emptyset}{d x}=q\left(n_{f}+n_{t}-p_{f}-p_{t}\right)
$$

The incident photon gets absorbed in the active material where it generates an exciton in the donor (PTB7). The diffusion of exciton takes place within the donor to the interface of Donor/Acceptor and gets dissociated into free electron and hole which are finally collected at their respective electrodes. The photocurrent is produced by connecting a load to the external circuit. Through simulations, variation in thickness of HTL, ETL and active layer is investigated and optimized for maximum PCE from the device.

\section{Materials}

PTB7 and $\mathrm{PC}_{71} \mathrm{BM}$ were procured from Oscilla (U.K.). AZO (99.99\%, $\mathrm{Al}_{2} \mathrm{O}_{3} / \mathrm{ZnO} 2 / 98$ wt\%) sputtering target was purchased from Stanford Advanced Materials (U.K.). Zinc acetate salt, $\mathrm{MoO}_{3}$ and $\mathrm{Ag}$ wire were purchased from Sigma Aldrich. For gold nanoflower synthesis, hydrogen tetrachloroaurate (III) trihydrate $\left(\mathrm{HAuCl}_{4} \cdot 3 \mathrm{H}_{2} \mathrm{O}\right)$ and L-ascorbic acid (L-AA) were procured from Sigma Aldrich. 


\section{Gold Nanoflowers Synthesis}

To synthesise gold nanoflowers, $0.01 \mathrm{M}$ gold salt $(100 \mu \mathrm{l})$ was added into $20 \mathrm{mM}$ ice-cold L-AA ( $35 \mathrm{ml})$ placed over a magnetic stirrer. The reaction was maintained at a constant temperature of $5{ }^{\circ} \mathrm{C}$ throughout. When gold salt was added, the colourless solution first turned purple and then changed to faint blue which confirmed the formation of gold nanoflowers. In this procedure, Au nanoflowers were synthesized by the self-assembly process in which nanoparticles with identical surface arrangements approach one another and combine together by oriented attachment. The gold ions present in the aqueous solution of hydrogen tetrachloroaurate (III) trihydrate salt are reduced by the ascorbate ions and stimulate nucleation for the synthesis of Au nanoflowers. Finally, these nanoparticles turned into nanoflowers through ostwald ripening process.

\section{Device Fabrication}

AZO cathode was sputtered on the pre-cleaned glass substrate utilizing if sputtering. It was then patterned through the laser scriber. The cleaning of the patterned AZO glass (sheet resistance around 20 $\Omega / \mathrm{sq}$ ) was done first in detergent, then in water, acetone and 2-propanol under sonication and finally dried using an oven[28]. Spin coating of a $\mathrm{ZnO}$ layer $(\sim 30 \mathrm{~nm})$ was done over AZO sputtered glass substrate at $3500 \mathrm{rpm}$ and subsequently annealed at $200{ }^{\circ} \mathrm{C}$ for $30 \mathrm{~min}[29]$. The PTB7:PC 71 BM photoactive layer ( 200 nm) having a blend ratio 1:1.5 was deposited over ZnO through spin-coating the solution containing chlorobenzene solvent $\left(25 \mathrm{mg} / \mathrm{ml}\right.$ )[30]. A $10 \mathrm{~nm}$ thick $\mathrm{MoO}_{3}$ (Molybdenum trioxide) film was thermally deposited over the photoactive layer[31]. Finally, the Ag anode of around $100 \mathrm{~nm}$ thickness was thermally coated in a high vacuum $\left(\sim 8 \times 10^{-6} \mathrm{mbar}\right)$ using a shadow mask. This completes our first device (AZO based device). Similarly, a second device (ITO based control device) with an identical structure based on ITO window cathode was also developed for comparison purposes. Finally, a third device (plasmonic assisted AZO based device) was developed using AZO as front cathode and introducing gold nanoflowers in the active layer blend. All these fabricated devices had an active area $\sim 4$ $\mathrm{mm}^{2}$.

\section{Characterization}

The absorption spectrum of the gold nanoflowers was recorded using EVOLUTION 300 UV-VIS (Thermo Scientific). Transmission electron microscopy (TEM) (JEOL-2100F, Japan) was performed to examine the shape and size of the gold nanoflowers. The X-ray diffraction data have been collected with a PANalytical diffractometer (EMPYREAN). The current-voltage (I-V) characteristic curve for the AZO film was recorded using the Keithley I-V setup. The optical spectra in transmission mode for ITO and AZO cathode while in absorbance mode for active layer (PTB7:PC 71 BM) were carried out using EVOLUTION 300 UV-VIS (Thermo Scientific). Raman mapping is done for AZO films, to confirm the uniformity, using a $514.5 \mathrm{~nm}$ argon ion laser excitation. The J-V curves of the OSCs were recorded through the source meter (Keithley 2450) with devices illuminated under AM1.5G $\left(100 \mathrm{~mW} / \mathrm{cm}^{2}\right)$ solar simulator. The external quantum efficiency (EQE) measurements were recorded with the help of a Tunable Light Source instrument 
(Newport) equipped with a standard silicon diode. The UV assisted acceleration ageing test was performed using a mercury vapour lamp having an intensity of $90 \mathrm{~mW} / \mathrm{cm}^{2}$.

\section{Results And Discussion}

\section{Device Optimization Through Simulations}

Optimization of Active Layer:- The thickness of the photoactive layer affects the incident light absorption and hence exciton generation in OSC, therefore its optimization is very crucial. To improve the PCE of the inverted OSC, the active layer was varied between 100-300 nm thickness[32]. Fig. 2 depicts the current density-voltage curves and the extracted electrical parameters for different active layer thicknesses. The $\mathrm{J}-\mathrm{V}$ characteristics exhibited sensitive relation with the active layer thickness since Jsc largely depends upon absorption inside the photoactive layer. It is observed that Jsc, Pmax, $\eta$ and FF show considerable dependence on the active layer thickness whereas Voc appears to be insensitive. The charge carrier generation and movement expressed by Jsc depends on the absorption and the diffusion length. For active layer thicknesses less than $200 \mathrm{~nm}$, the absorption is incomplete whereas thickness above $200 \mathrm{~nm}$ enhances the exciton recombination and hence reduces the charge carrier collection. In view of this, the active layer was optimized to be $200 \mathrm{~nm}$. For the $200 \mathrm{~nm}$ thick active layer, $P_{\max }$ and PCE of $62.82 \mathrm{~W} / \mathrm{m}^{2}$ and $6.28 \%$ respectively have been obtained through simulations. Also, FF falls rapidly with an increase in active layer thickness while $\mathrm{V}_{\text {oc }}$ falls slightly from $0.62 \mathrm{~V}$ to $0.60 \mathrm{~V}$. The decrease in FF is because of the enhanced series resistance. The simulated results show the maximum efficiency for the $200 \mathrm{~nm}$ thick active layer, hence it was selected to fabricate the actual device.

Optimization of ETL:- The electron transport layer commonly known as the buffer layer is used to increase carrier collection in cells. This study shows the dependence of the performance of simulated inverted OSC on the variation in ETL thickness. The photoactive layer PTB7:PC ${ }_{71} B M$ is fixed at the optimized thickness of $200 \mathrm{~nm}$. The ETL material between the front cathode (AZO) and the photoactive layer (PTB7:PC $\left.{ }_{71} B M\right)$ should favour the electron conduction and restrict the transport of holes toward the cathode, thereby, preventing the recombination of charge carriers near the cathode. The alignment of energy levels for the active layer, ETL and cathode clearly shows that ZnO having a large bandgap $\left(E_{g}\right)$ and electron affinity similar to AZO is the most suitable material for ETL. Zinc oxide (ZnO) is chosen as ETL and its thickness is varied between $10-50 \mathrm{~nm}$. Fig. 3 depicts the observed $\mathrm{J}-\mathrm{V}$ characteristics and the electrical parameters extracted. The variations of $\mathrm{J}_{\mathrm{sc}}, \mathrm{P}_{\max }$ and PCE show a similar trend with the increase in ETL thickness. Also, the increase in ETL thickness has only minimal effect on all the parameters in our simulated device. The $\mathrm{V}_{\mathrm{oc}}$ and FF remain almost constant with variations in $\mathrm{ETL}$ thickness[33]. Overall, it can be concluded that ETL thickness slightly affects the performance parameters of the OSC and the optimized thickness for ETL observed to be $30 \mathrm{~nm}$. 
Optimization of HTL:- From the simulations results of the preceding sections, the thickness of ETL and active layer is optimized to $30 \mathrm{~nm}$ and $200 \mathrm{~nm}$ respectively. In this section, the variation of HTL thickness is investigated through simulations. $\mathrm{MoO}_{3}$ is chosen as $\mathrm{HTL}$ due to its concomitant properties like better transparency, high conductivity and better hole transportation[34]. The $\mathrm{MoO}_{3}$ thickness is varied between 5-15 $\mathrm{nm}$. The J-V characteristic curves and other parameters obtained through the simulations are drawn in Fig. 4 (a) and Fig. 4 (b) respectively. As seen in Fig. 4(b), the variation in HTL thickness has no prominent effect on $\mathrm{FF}$ and $\mathrm{V}_{\mathrm{OC}}$, which is due to the low hole mobility. The optimized inverted organic solar cell is obtained at $10 \mathrm{~nm}$ thickness of $\mathrm{HTL}$. $\mathrm{J}_{\mathrm{sC}}, \mathrm{P}_{\max }$ and PCE slightly vary with variation in the thickness of HTL and exhibit the optimum values at $10 \mathrm{~nm}$. FF and Voc remain almost insensitive to variation in thickness of HTL. Consequently, the thickness of HTL has a minor effect on electrical parameters of simulated inverted OSC due to the low hole mobility[35].

\section{Cathode Investigation}

As the aim of this work is to deposit AZO as TCO having comparable characteristics to ITO, we started with deposition and characterization of AZO first. The AZO film over glass substrate was deposited using rf sputtering and the AZO film thickness was determined to be $800 \mathrm{~nm}$ with the help of Dektak profilometer. The XRD pattern for the AZO cathode film is shown in Fig. 5 (a). The AZO crystallites exhibit a hexagonal wurtzite structure (ICDD-00-051-0037). The spectra show a major peak at $62.85^{\circ}$ reflecting the lateral growth along the crystallographic axis (103). Other peaks at $34.28^{\circ}, 36.18^{\circ}, 47.39^{\circ}$ and $56.41^{\circ}$ with small intensity are detected at axes (002), (101), (102) and (110), respectively. The (002) plane is related to vertical growth, however, the presence of a relatively intense peak along (103) is indicative of non-vertical growth. Another critical parameter of TCO is the low sheet resistance, which corresponds to better conductivity. The sheet resistance of AZO film was calculated using a four-point probe. Fig. 5 (b) shows the room temperature I-V curve, with sheet resistance around $20 \Omega$ /sq which approaches the sheet resistance of ITO electrode $\otimes 15 \Omega /$ sq.

The UV-Vis spectra in transmission mode for AZO and ITO films were measured and the wavelength dependent transmittance for both the films are plotted Fig. 5(c). It is evident from the plot that AZO film has an average transmittance of around $80 \%$ in the visible region ( $400 \mathrm{~nm}$ to $800 \mathrm{~nm}$ ), comparable to ITO/glass. Also, the transmittance curve of AZO depicts a cut off at the wavelength $~ 380 \mathrm{~nm}$ which is due to the UV absorption properties of AZO (bandgap $3.26 \mathrm{eV}$ ). Although AZO blocks the UV part of the solar spectrum, most of the sunlight passes through it as the major portion of the solar radiation lies in the spectrum having $\lambda>380 \mathrm{~nm}$, hence enabling efficient absorption in the device. This UV filtering capacity of the AZO film enhances the stability of the device by preventing device degradation due to UV exposure. Uniformity is a prerequisite for the cathode of a solar cell. To ensure the uniformity in AZO cathode, raman mapping was done at various locations over the film. Fig. 5(d) depicts the optical microscopic image of the film in the inset, indicating six different points where raman mapping was performed. The AZO characteristic anomalous peaks at 274,475 and $511 \mathrm{~cm}^{-1}$ were present and for all the locations similar spectra were recorded, indicative of uniformity of the AZO cathode[36]. Since Al- 
doped zinc oxide has demonstrated electrical and optical properties that are comparable to ITO, it can effectively replace ITO to achieve cost-effective and stable photovoltaic devices.

\section{Gold Nanoflowers Characterization}

Efficient light absorption in inverted OSCs is important for their better performance and the inclusion of gold nanoflowers in the active layer serves the purpose without compromising their architecture. The incorporated plasmonic nanoparticles $(5-50 \mathrm{~nm})$ act like antennas and the incoming light energy is stored in LSPR modes resulting in multiple times enhancement of the local electromagnetic field leading to enhanced light absorption. The increase in exciton dissociation is also possible through interactions between the plasmons and the excitons thereby lowering the recombination loss of excitons. The introduction of the gold nanoflowers inside the active layer improves the PCE of the OSCs using the optical property of LSPR without affecting their electrical characteristics. Gold is preferably used as it is highly resistive to oxidation and hence ensures a longer lifetime of the cell. The size and shape of nanoflowers were investigated using TEM apparatus. The TEM picture shown in Fig. 6(a) suggests the formation of gold nanoflowers. The average size for gold nanoflowers analyzed by ImageJ software was $33 \mathrm{~nm}$. The core of the nanoflowers was around $25 \mathrm{~nm}$ with the length of each petal around $4 \mathrm{~nm}$. The absorption spectrum for the gold nanoflowers is the convolution of two absorption peaks as seen from fig. 6(b). There exist two absorption peaks within the visible regime, attributed to the surface plasmons in the gold nanoflowers. These nanoparticles show surface plasmon peaks at two different frequencies, the first peak at $588 \mathrm{~nm}$ arises from the core of nanoflowers and the other peak at $748 \mathrm{~nm}$ is due to free electrons present in petals.

\section{Device Characterization}

The simulation results of section 4.1 provided the optimized thickness of ETL ( $\mathrm{ZnO})$, active layer (PTB7:PC $\left.{ }_{71} \mathrm{BM}\right)$ and $\mathrm{HTL}\left(\mathrm{MoO}_{3}\right)$ to be $30 \mathrm{~nm}, 200 \mathrm{~nm}$ and $10 \mathrm{~nm}$ respectively. Utilizing these optimized thicknesses, we fabricated the three devices:- (1) AZO based device. (2) ITO based control device. (3) plasmonic assisted AZO based device. The first AZO based device is fabricated based on the device geometry AZO/ZnO/PTB7:PC ${ }_{71} \mathrm{BM} / \mathrm{MoO}_{3} / \mathrm{Ag}$. The second device contains ITO as a front cathode keeping the remaining structure identical and is used for comparison studies (control device). The third device consists of plasmonic gold nanoflowers (average size of $33 \mathrm{~nm}$ ) embedded into the active layer and using $\mathrm{AZO}$ as the front transparent conducting cathode. The $\mathrm{J}-\mathrm{V}$ measurements for all three fabricated inverted OSCs were recorded using $100 \mathrm{~mW} / \mathrm{cm}^{2}$ AM $1.5 \mathrm{G}$ and it was observed that AZO based device showed a PCE of $6.19 \%$ which was slightly less than PCE $6.83 \%$ for the ITO based control device. Also, the PCE of the AZO based OSC was further enhanced to $7.01 \%$ when gold nanoflowers were embedded within the active layer of the OSC. The J-V characteristic curves for the three fabricated cells are shown in Fig. 7(a) and the extracted important parameters like PCE, FF, $P_{\text {max }}, V_{O C}$ and $J_{S C}$ are included in Table 1. Also, the parameters extracted from the J-V curves of the experimentally fabricated AZO based inverted OSC matches well with the theoretically optimized device using GPVDM simulation which ensures the credibility of the simulation results. The EQE measurements for the experimentally 
fabricated cells are depicted in Fig. 7(b) and it is evident that the cell with AZO front transparent cathode shows a red-shift in the short wavelength region associated with cut off in transmittance $(\lambda<380 \mathrm{~nm})$ for AZO cathode. The EQE curve for the AZO based device follows the absorbance in the visible portion of the solar spectrum indicating the non-contribution of the UV in the measured $\mathrm{J}_{\mathrm{SC}}$. This can be attributed to the UV blocking property of $\mathrm{AZO}$ and is further augmented by the use of $\mathrm{ZnO}$ as ETL. It is interesting that $A Z O$ is acting as a transparent cathode and blocks the UV, which can increase the lifetime of the fabricated OSC.

Upon introduction of gold nanoflowers inside the active region, there is a considerable enhancement in EQE in the wavelength regime $400-780 \mathrm{~nm}$ for the AZO cathode based device. This enhancement in EQE is due to near field enhancement shown by plasmonic gold nanoflowers which increases absorption inside the active layer. Fig. 7(c) reports the absorbance spectra of the PTB7, PC ${ }_{71} B M$ and PTB7:PC 71 BM for the wavelength range $330-800 \mathrm{~nm}$. PTB7 exhibits stronger light absorption ranging from 550 to 750 $\mathrm{nm}$ while $\mathrm{PC}_{71} \mathrm{BM}$ presents an overwhelming absorption of light in the wavelength range 330 to $550 \mathrm{~nm}$. Therefore, $\mathrm{PC}_{71} \mathrm{BM}$ provides complimentary light absorption when mixed with PTB7 and the blend becomes a champion active layer material.

The OSC degradation due to UV exposure can be eliminated by restricting the UV portion from the solar spectrum to reach the photoactive layer and this can be effectively done by the AZO front cathode. It is clear that AZO film has very low transmittance in the UV region showing its potential candidature as a UV filtering layer. The UV assisted acceleration ageing test was done to investigate the stability of the AZO based and ITO based inverted OSCs[37]. Both the devices with different front cathodes were illuminated under UV irradiation for an exposure time of 0 to $30 \mathrm{~min}$ from the cathode side. This test was performed inside the nitrogen filled glove box which

Table 1. Comparison of electrical parameters for the simulated and the fabricated devices.

\begin{tabular}{|lllll|}
\hline $\begin{array}{l}\text { Electrical } \\
\text { Parameters }\end{array}$ & $\begin{array}{l}\text { GPVDM } \\
\text { Simulated AZO } \\
\text { based device }\end{array}$ & $\begin{array}{l}\text { Fabricated ITO } \\
\text { based control } \\
\text { device }\end{array}$ & $\begin{array}{l}\text { Fabricated } \\
\text { AZO based } \\
\text { device }\end{array}$ & $\begin{array}{l}\text { Fabricated plasmonics } \\
\text { assisted AZO based } \\
\text { device }\end{array}$ \\
\hline $\mathrm{V}_{\mathrm{OC}}(\mathrm{V})$ & 0.61 & 0.62 & 0.60 & 0.61 \\
\hline $\mathrm{J}_{\mathrm{SC}}\left(\mathrm{mA} / \mathrm{cm}^{2}\right)$ & 15.21 & 16.10 & 15.20 & 16.15 \\
\hline $\mathrm{P}_{\mathrm{MAX}}\left(\mathrm{W} / \mathrm{m}^{2}\right)$ & 62.82 & 68.30 & 61.92 & 70.12 \\
\hline Fill Factor$(\%)$ & 67.71 & 68.42 & 67.89 & 71.18 \\
\hline $\mathrm{PCE}(\%)$ & 6.28 & 6.83 & 6.19 & 7.01 \\
\hline
\end{tabular}

neglects the possible degradation of OSC due to moisture and oxygen. Fig. 7 shows the change in device parameters (PCE, FF, $\mathrm{V}_{\mathrm{OC}}, \mathrm{J}_{\mathrm{SC}}$ ) recorded for the AZO and ITO (control) based inverted OSC under UV 
exposure of 0 to $30 \mathrm{~min}$. Though ITO based control device possesses slightly higher efficiency initially, a fast decrease in all important device parameters is observed from the graphs in Fig. 8. For the ITO based control device, parameters like PCE, $F F, V_{O C}$ and $J_{S C}$ dropped from $6.83 \%, 68.42 \%, 0.62 \mathrm{~V}, 16.10 \mathrm{~mA} / \mathrm{cm}^{2}$ to $3.08 \%, 47.23 \%, 0.46 \mathrm{~V}, 14.20 \mathrm{~mA} / \mathrm{cm}^{2}$ after $30 \mathrm{~min}$ of UV exposure. However, AZO based device exhibits only a slight decrease in the device performance parameters under the same test and hence ensures better stability. For the AZO based inverted OSC, the important parameters including PCE, FF, $\mathrm{V}_{\mathrm{OC}}$ and $\mathrm{J}_{\mathrm{SC}}$ changed from $6.19 \%, 67.89 \%, 0.60 \mathrm{~V}, 15.20 \mathrm{~mA} / \mathrm{cm}^{2}$ to $5.48 \%, 64 \%, 0.57 \mathrm{~V}, 15.02 \mathrm{~mA} / \mathrm{cm}^{2}$ after 30 min of UV exposure. This study reveals the better stability of AZO based OSC in comparison to ITO based devices and this is because of the UV blocking properties of the AZO layer. This work clearly indicates that AZO can effectively replace ITO cathode in OSCs to enhance the lifetime of the device.

\section{Conclusions}

In this work, ITO free inverted organic solar cell has been simulated and optimized using GPVDM software and the optimized device is fabricated experimentally along with a control device for comparative studies. The dependence of active layer, ETL and HTL thickness on device performance is investigated and the results can be concluded as: the active layer thickness has a significant effect, the ETL thickness is almost insensitive and the HTL thickness has minor effects on the performance of the proposed geometry. The best performing device consists of $30 \mathrm{~nm} \mathrm{ZnO,} 200 \mathrm{~nm}$ PTB7:PC ${ }_{71} \mathrm{BM}$ and 10 $\mathrm{nm} \mathrm{MoO}$ thickness. The fabricated AZO based inverted OSC shows better stability than the ITO based control device as suggested by the UV assisted acceleration ageing test. Upon UV exposure, a fast degradation in all the performing parameters is observed for the ITO based control device while only moderate degradation is there for AZO based OSC. The enhanced lifetime of the AZO based OSC is due to UV blocking behaviour shown by the AZO cathode layer. The efficiency of the AZO based device is further enhanced to $7.01 \%$ when Au nanoflowers are embedded inside the active region of the device. This PCE enhancement is attributed to the plasmonic effect associated with the gold nanoflowers. Overall, this work leads to the development of a cost-effective, efficient and stable ITO free inverted OSC.

\section{Abbreviations}

OSC: Organic solar cell; ITO: Indium tin oxide; AZO: Aluminium doped zinc oxide; LSPR: Localized surface plasmon resonance; ETL: Electron transport layer; HTL: Hole transport layer; PCE: Power conversion efficiency; GPVDM: General purpose photovoltaic device model; PTB7: Poly [[4,8-bis[(2ethylhexyl)oxy]benzo[1,2-b:4,5-b']dithiophene-2,6-diyl][3-fluoro-2-[(2-ethylhexyl)carbonyl]thieno[3,4b]thiophenediyl]]; $\mathrm{PC}_{71} \mathrm{BM}$ : [6,6]-phenyl $\mathrm{C}_{71}$ butyric acid methyl ester; L-AA: L-ascorbic acid; $\mathrm{MoO}_{3}$ : Molybdenum trioxide

\section{Declarations}

Acknowledgements 
This work presented in this paper is funded by the Department of Science and Technology (DST), SERB sanction order no. EEQ/2017/000801.

\section{Competing Interests}

The authors have no known competing financial interests to declare.

\section{References}

1. S. A, K. R. A, Das BC (2020) Air-processed active-layer of organic solar cells investigated by conducting AFM for precise defect detection. RSC Advances 10:24882-24892. https://doi.org/10.1039/d0ra03986a

2. Mellit A (2020) Recent Applications of Artificial Intelligence in Fault Diagnosis of Photovoltaic Systems

3. Duan X, Zhang X, Zhang Y (2018) High Performance Organic-Nanostructured Silicon Hybrid Solar Cell with Modified Surface Structure. Nanoscale Research Letters 13:. https://doi.org/10.1186/s11671-018-2703-2

4. Wang K, Liu C, Meng T, et al (2016) Inverted organic photovoltaic cells. Chemical Society Reviews 45:2937-2975. https://doi.org/10.1039/c5cs00831j

5. Günes S, Marjanovic N, Nedeljkovic JM, Sariciftci NS (2008) Photovoltaic characterization of hybrid solar cells using surface modified TiO2 nanoparticles and poly(3-hexyl)thiophene. Nanotechnology 19:. https://doi.org/10.1088/0957-4484/19/42/424009

6. Ganesan S, Mehta S, Gupta D (2019) Fully printed organic solar cells - A review of techniques, challenges and their solutions. Opto-electronics Review 27:298-320. https://doi.org/10.1016/j.opelre.2019.09.002

7. Bauer A, Wahl T, Hanisch J, Ahlswede E (2012) ZnO:Al cathode for highly efficient, semitransparent 4 organic solar cells utilizing TiO $x$ and aluminum interlayers. Applied Physics Letters 100:2012-2015. https://doi.org/10.1063/1.3685718

8. Maache M, Devers T, Chala A (2017) Al-doped and pure ZnO thin films elaborated by sol-gel spin coating process for optoelectronic applications Al-doped and pure $\mathrm{ZnO}$ thin films elaborated by solgel spin coating process for optoelectronic applications. https://doi.org/10.21883/FTP.2017.12.45182.8078

9. Shi T, Zhu X, Tu G (2014) Efficient inverted polymer solar cells based on ultrathin aluminum interlayer modified aluminum-doped zinc oxide electrode. Applied Physics Letters 104:10-14. https://doi.org/10.1063/1.4868101

10. Berǹde JC, Berredjem Y, Cattin L, Morsli M (2008) Improvement of organic solar cell performances using a zinc oxide anode coated by an ultrathin metallic layer. Applied Physics Letters 92:37-40. https://doi.org/10.1063/1.2888176 
11. Sun L, Zeng W, Xie C, et al (2020) Flexible All-Solution-Processed Organic Solar Cells with HighPerformance Nonfullerene Active Layers. Advanced Materials 32:1-8. https://doi.org/10.1002/adma.201907840

12. Liu S, Yuan J, Deng W, et al (2020) High-efficiency organic solar cells with low non-radiative recombination loss and low energetic disorder. Nature Photonics 14:300-305. https://doi.org/10.1038/s41566-019-0573-5

13. Chauhan M, Sharma A, Patel J, et al (2018) Investigating the influence of charge transport on the performance of PTB7:PC71BM based organic solar cells. Physical Chemistry Chemical Physics 20:17304-17312. https://doi.org/10.1039/c8cp02730g

14. Wang J, Jia S, Cao Y, et al (2018) Design Principles for Nanoparticle Plasmon-Enhanced Organic Solar Cells. Nanoscale Research Letters 13

15. Qiao L, Wang D, Zuo L, et al (2011) Localized surface plasmon resonance enhanced organic solar cell with gold nanospheres. Applied Energy 88:848-852. https://doi.org/10.1016/j.apenergy.2010.09.021

16. Cells S (2011) Surface Plasmonic E ff ects of Metallic Nanoparticles on the Performance of Polymer Bulk Heterojunction. 959-967

17. Patel AS, Juneja S, Kanaujia PK, et al (2018) Gold nanoflowers as efficient hosts for SERS based sensing and bio-imaging. Nano-Structures and Nano-Objects 16:329-336. https://doi.org/10.1016/j.nanoso.2018.09.001

18. Damena KL (2019) Investigation of Organic Solar Cell at different Active Layer Thickness and Suns using GPVDM. 1615-1626

19. Aprilia A, Wulandari P, Suendo V, et al (2013) Solar Energy Materials \& Solar Cells Influences of dopant concentration in sol - gel derived AZO layer on the performance of P3HT : PCBM based inverted solar cell. Solar Energy Materials and Solar Cells 111:181-188. https://doi.org/10.1016/j.solmat.2012.12.033

20. Frankenstein H, Leng CZ, Losego MD, Frey GL (2019) Atomic layer deposition of ZnO electron transporting layers directly onto the active layer of organic solar cells. Organic Electronics 64:37-46. https://doi.org/10.1016/j.orgel.2018.10.002

21. Pali LS, Gupta SK, Garg A (2018) Organic solar cells on Al electroded opaque substrates: Assessing the need of ZnO as electron transport layer. Solar Energy 160:396-403. https://doi.org/10.1016/j.solener.2017.12.028

22. Minami T (2008) Substitution of transparent conducting oxide thin films for indium tin oxide transparent electrode applications. Thin Solid Films 516:1314-1321. https://doi.org/10.1016/j.tsf.2007.03.082

23. Ma W, Yang C, Gong X, et al (2005) Thermally stable, efficient polymer solar cells with nanoscale control of the interpenetrating network morphology. Advanced Functional Materials 15:1617-1622. https://doi.org/10.1002/adfm.200500211 
24. He Y, Liu C, Li J, et al (2015) Improved Power Conversion Efficiency of Inverted Organic Solar Cells by Incorporating Au Nanorods into Active Layer. ACS Applied Materials and Interfaces 7:15848-15854. https://doi.org/10.1021/acsami.5b03024

25. Abdallaoui M, Sengouga N, Chala A, et al (2020) Comparative study of conventional and inverted P3HT: PCBM organic solar cell. Optical Materials 105:109916. https://doi.org/10.1016/j.optmat.2020.109916

26. Farooq W, Khan AD, Khan AD, Noman M (2020) Enhancing the power conversion efficiency of organic solar cells. Optik 208:. https://doi.org/10.1016/j.ijleo.2019.164093

27. Chakraborty K, Malakar S, Mandal DK, et al (2019) Experimental Prediction of Effect of Thickness of Active Layer of Photovoltaic Device on a series of Electrical Parameters using GPVDM Software. International Journal of Advanced Science and Engineering 6:42-46. https://doi.org/10.29294/ijase.6.s1.2019.42-46

28. Chen D, Zhang C, Wang Z, et al (2014) High efficient ITO free inverted organic solar cells based on ultrathin Ca modified AZO cathode and their light soaking issue. ORGANIC ELECTRONICS 15:30063015. https://doi.org/10.1016/j.orgel.2014.08.042

29. Dong JJ, Wu J, Hao HY, et al (2017) Synthesis of ZnO Nanocrystals and Application in Inverted Polymer Solar Cells. Nanoscale Research Letters 12:. https://doi.org/10.1186/s11671-017-2283-6

30. Das S, Keum JK, Browning JF, et al (2015) Correlating high power conversion efficiency of PTB7:PC71BM inverted organic solar cells with nanoscale structures. Nanoscale 7:15576-15583. https://doi.org/10.1039/c5nr03332b

31. Morvillo P, Parenti F, Diana R, et al (2012) A novel copolymer from benzodithiophene and alkylsulfanyl-bithiophene: Synthesis, characterization and application in polymer solar cells. Solar Energy Materials and Solar Cells 104:45-52. https://doi.org/10.1016/j.solmat.2012.04.044

32. Sharma N, Gupta SK, Singh Negi CM (2019) Influence of active layer thickness on photovoltaic performance of PTB7:PC70BM bulk heterojunction solar cell. Superlattices and Microstructures 135:106278. https://doi.org/10.1016/j.spmi.2019.106278

33. Luo J, Wang Y, Zhang Q (2018) Progress in perovskite solar cells based on ZnO nanostructures. Solar Energy 163:289-306. https://doi.org/10.1016/j.solener.2018.01.035

34. Nair SS, Kumar D (2016) Morphology control in the bulk heterojunction blend of inverted organic solar cell via co-solvent addition. Advanced Materials Letters 7:806-812. https://doi.org/10.5185/amlett.2016.6204

35. Li P, Wu B, Yang YC, et al (2018) Improved charge transport ability of polymer solar cells by using NPB/MoO3 as anode buffer layer. Solar Energy 170:212-216. https://doi.org/10.1016/j.solener.2018.04.020

36. Mohanty BC, Kim BK, Yeon DH, et al (2011) Structural and Raman Scattering Properties of ZnO:Al Thin Films Sputter-Deposited at Room Temperature. Journal of The Electrochemical Society 159:H96-H101. https://doi.org/10.1149/2.027202jes 
37. Liu H, Wu Z, Hu J, et al (2013) Efficient and ultraviolet durable inverted organic solar cells based on an aluminum-doped zinc oxide transparent cathode. Applied Physics Letters 103:. https://doi.org/10.1063/1.4816786

\section{Figures}

(a)

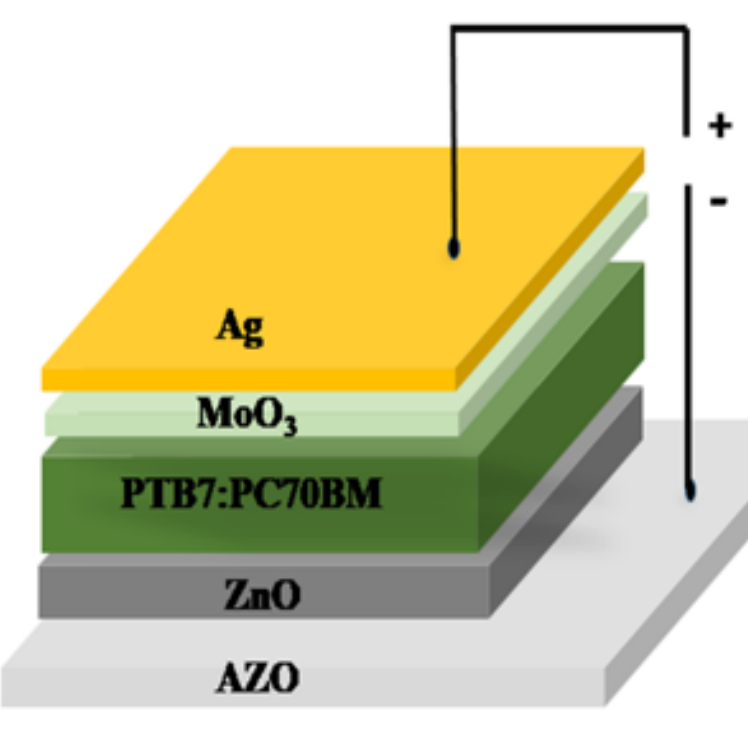

(b)

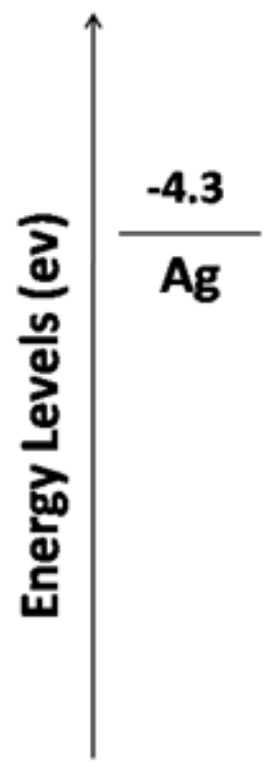

$-2.3$

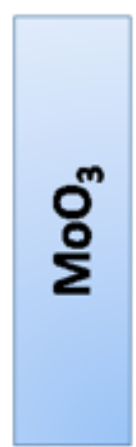

$-5.3$

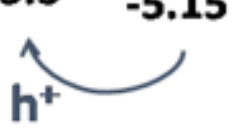

$-6.1$
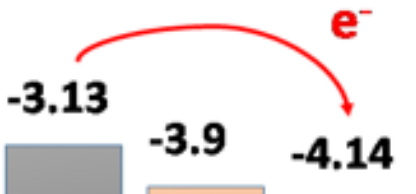

$-4.75$

AZO

Figure 1

(a) ITO free inverted OSC geometry. (b) Energy level diagram for the device.

(a)

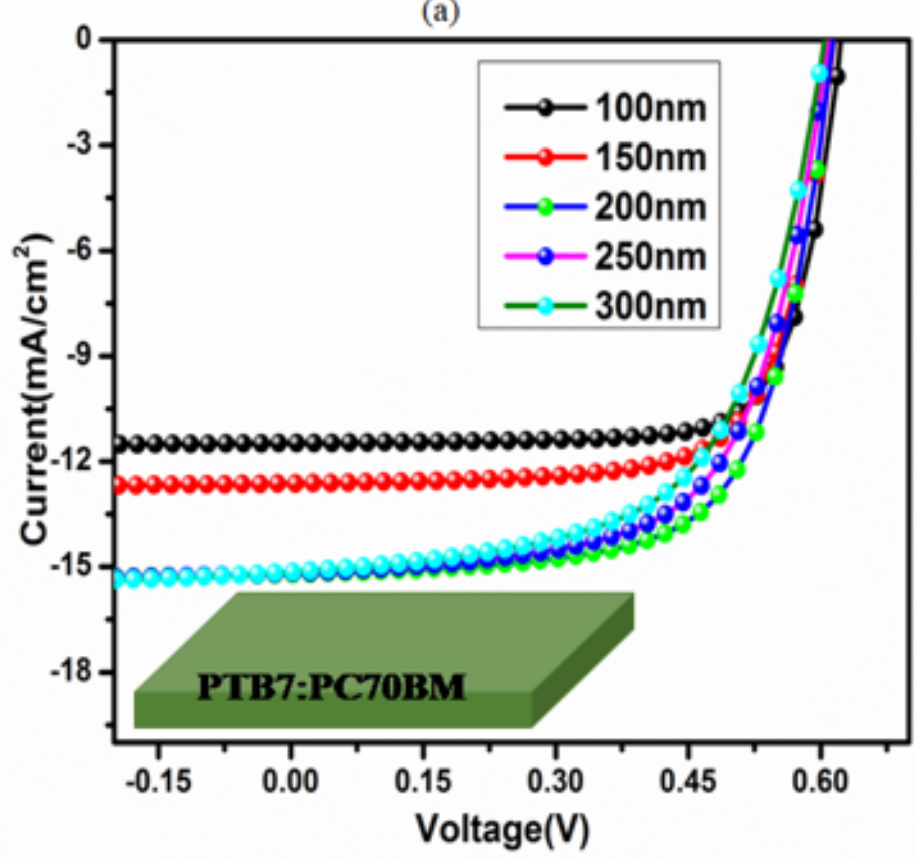

(b)

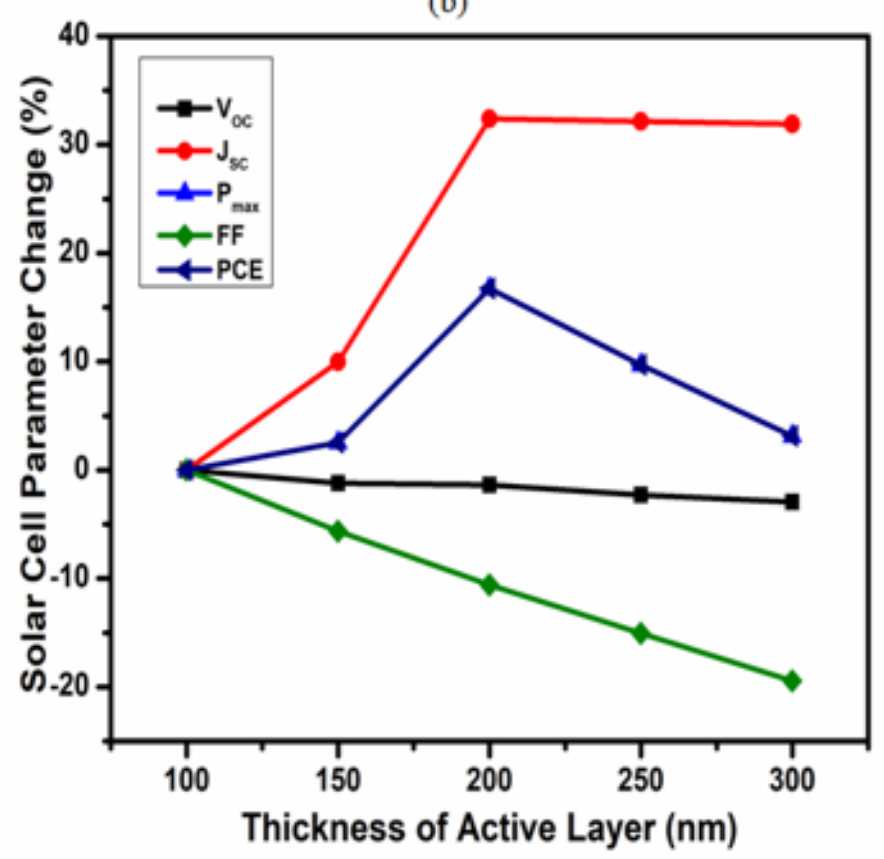

Figure 2 
(a) J-V characteristic curves for various thicknesses of the active layer. (b) Extracted electrical parameters change vs active layer thickness plot.

(a)

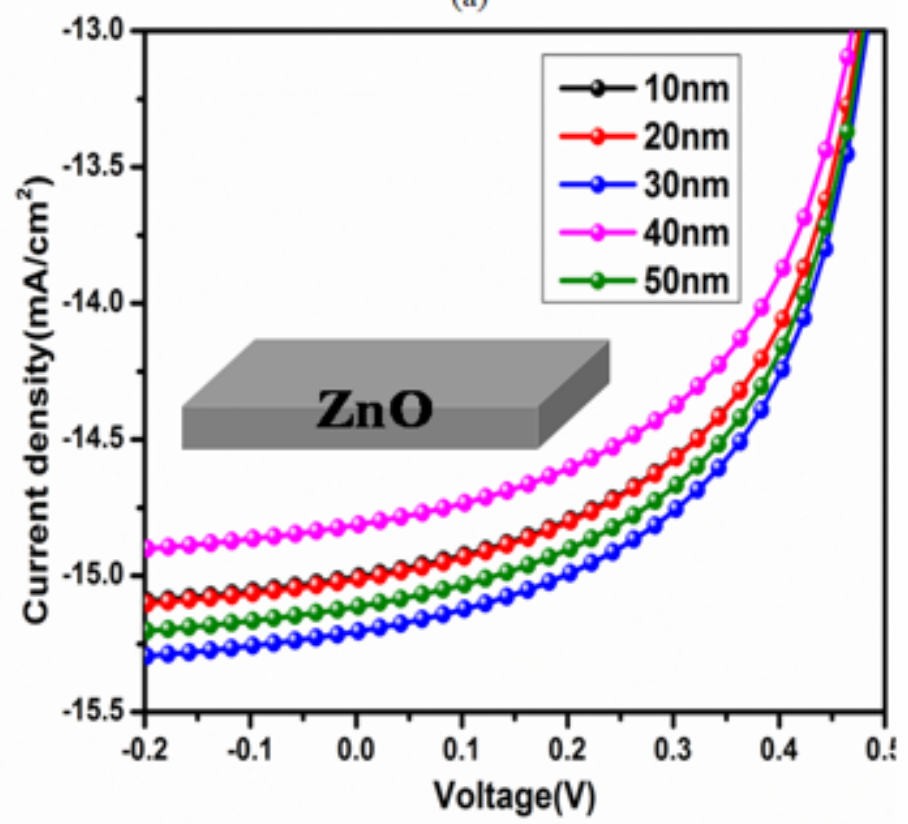

(b)

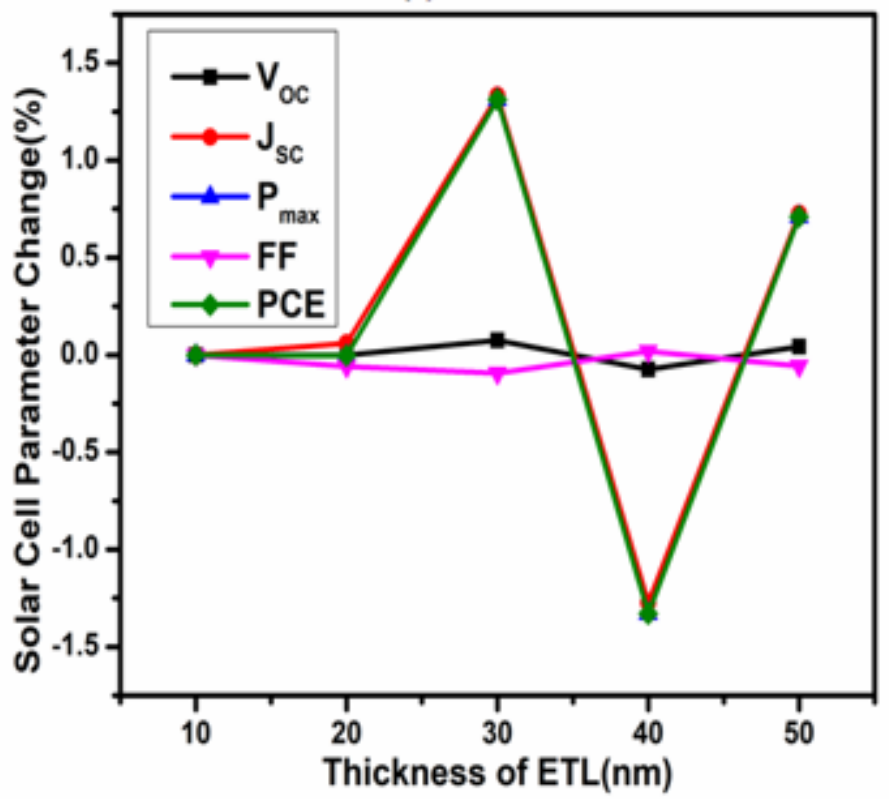

Figure 3

(a) J-V characteristic curves for different ETL thicknesses. (b) Extracted electrical parameters change vs ETL thickness plot.
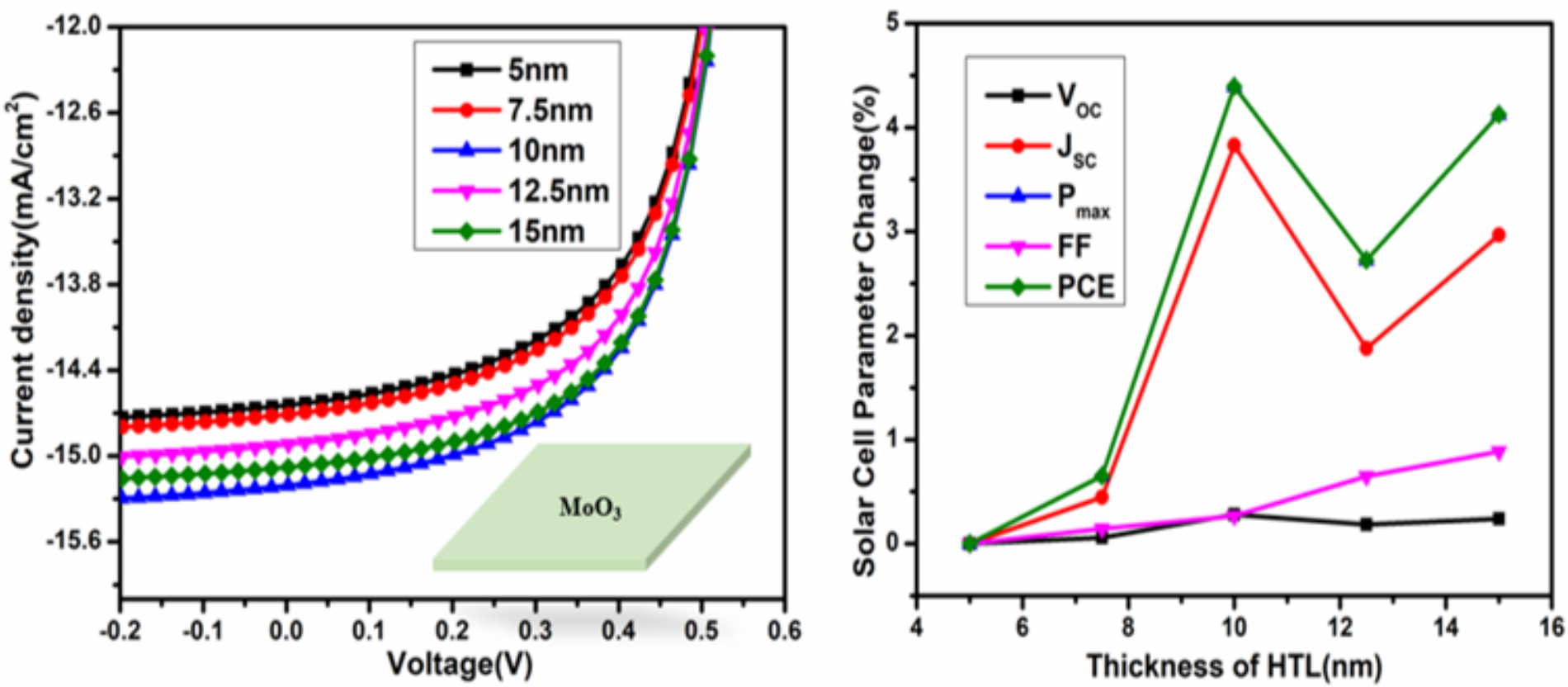

Figure 4 
(a) J-V characteristic curves for various HTL thicknesses. (b) Extracted electrical parameters change vs HTL thickness plot.

(a)

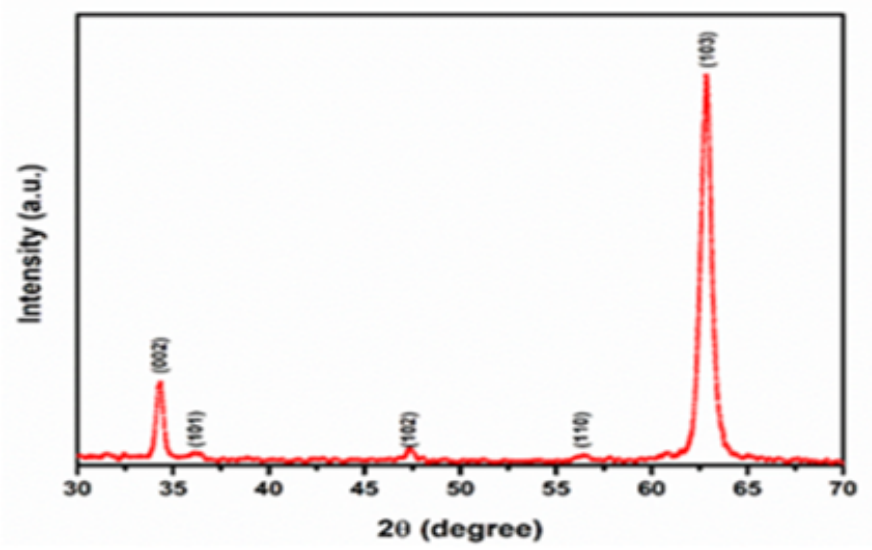

(c)

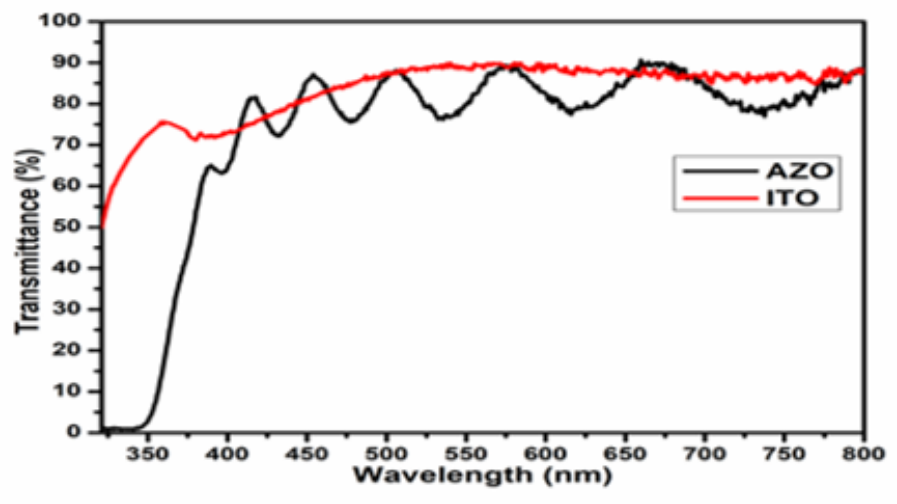

(b)

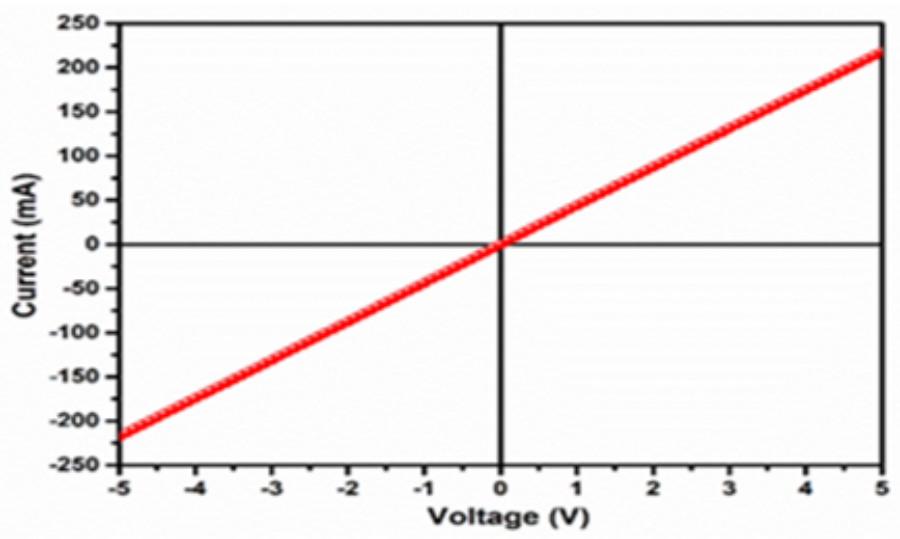

(d)

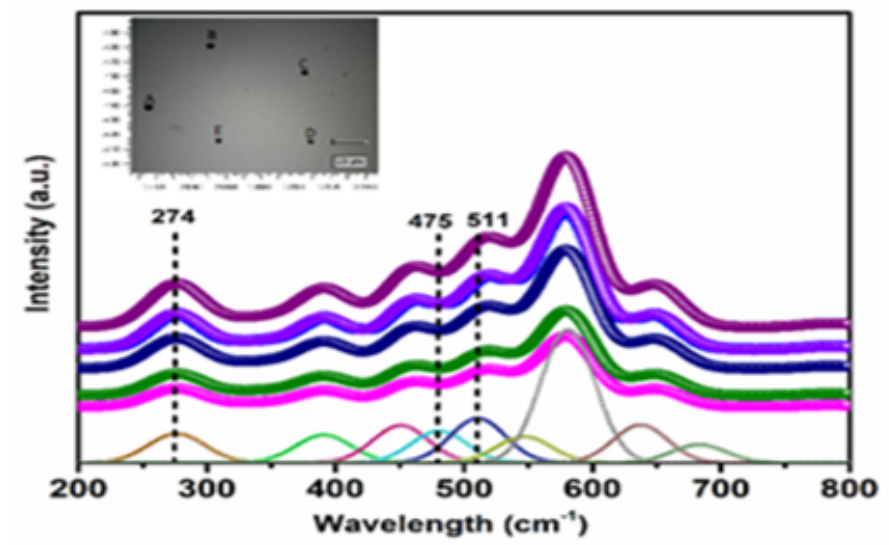

Figure 5

For AZO/ glass (a) XRD pattern (b) I-V measurement (c) Transparency measurement and (d) Raman spectra. 

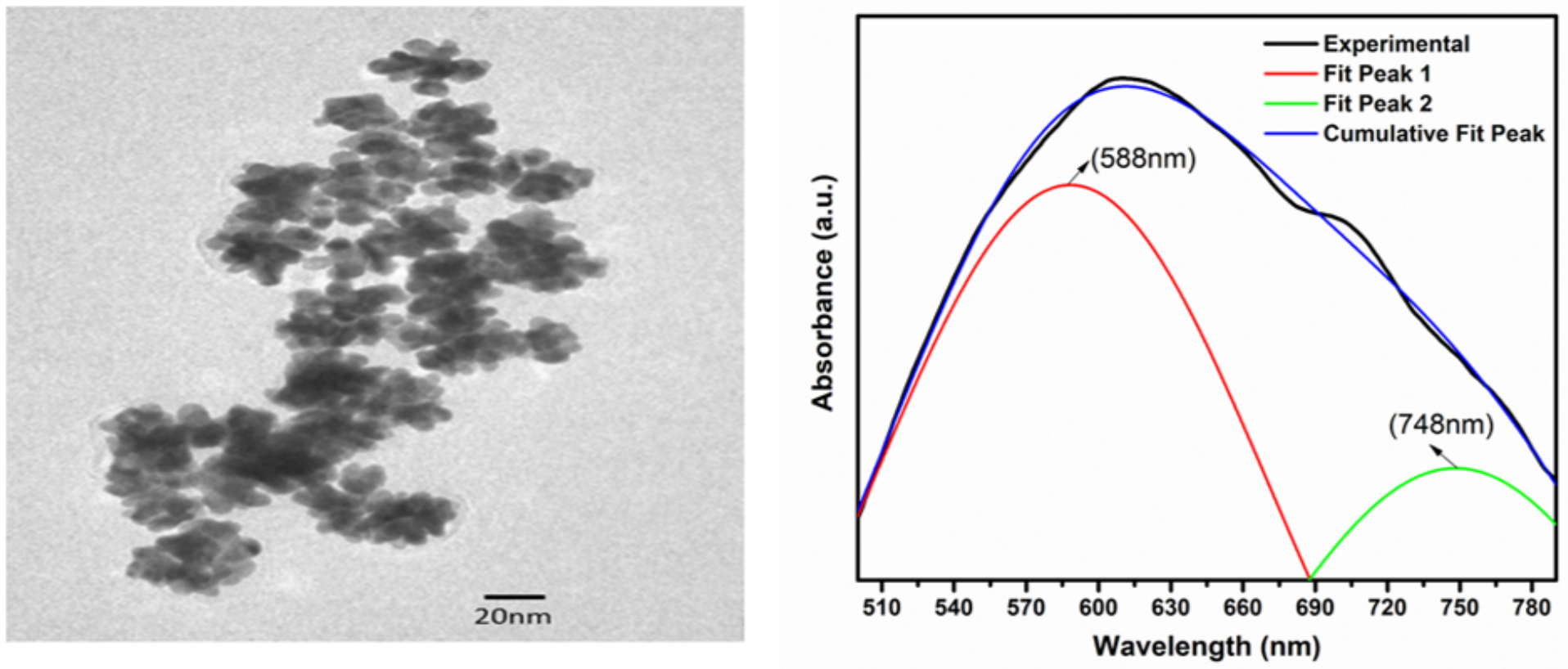

Figure 6

For synthesized gold nanoflowers (a) TEM image. (b) UV-Visible absorption spectrum. 
(a)

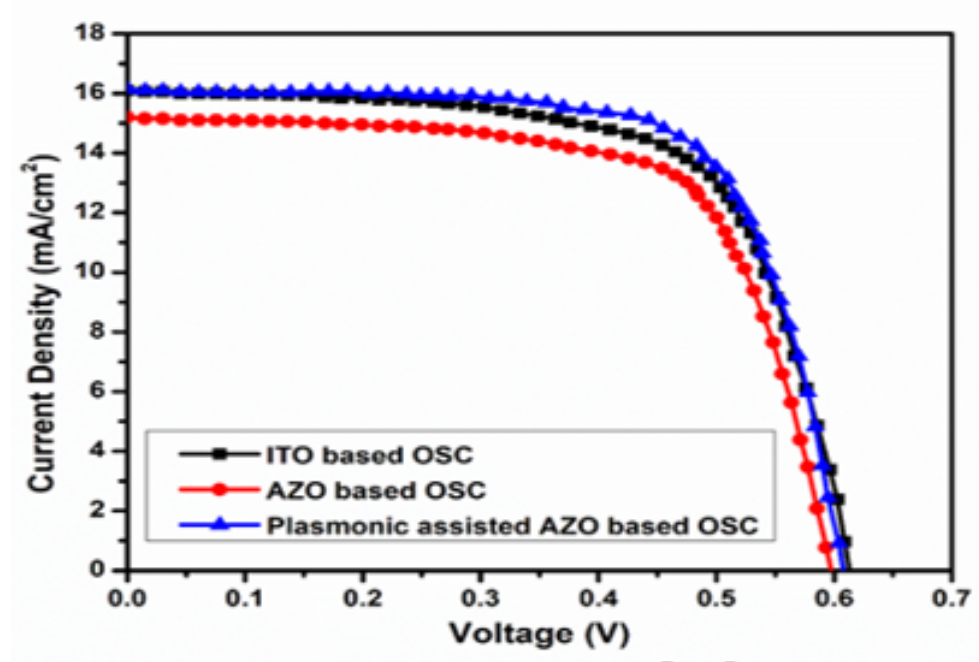

(c)
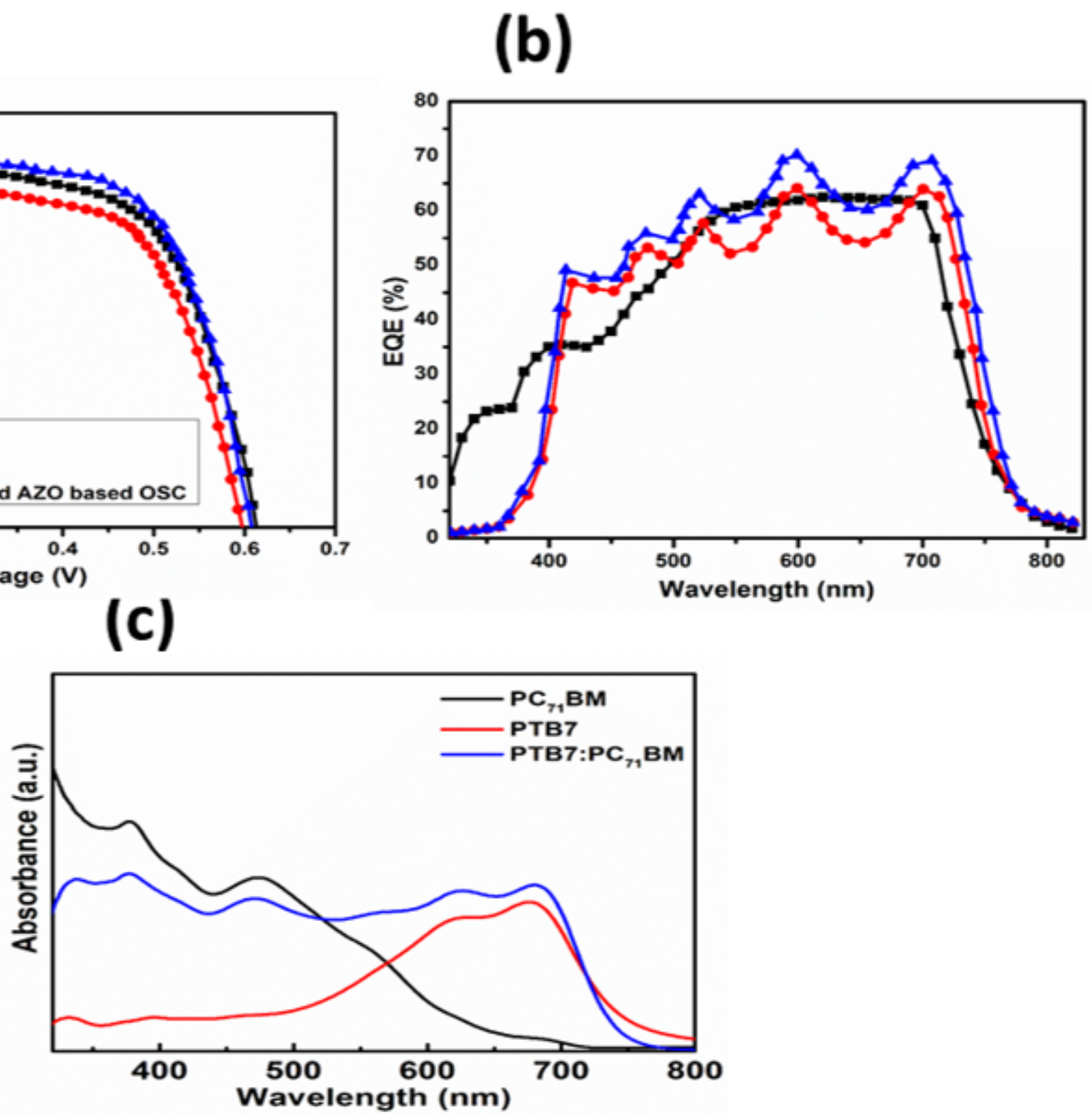

Figure 7

(a) J-V curves of the fabricated OSCs:- ITO based device (black), AZO based device (red) and plasmonic assisted AZO based device (blue). (b) EQE measurements of the corresponding OSCs. (c) absorption curves of the active layer. 

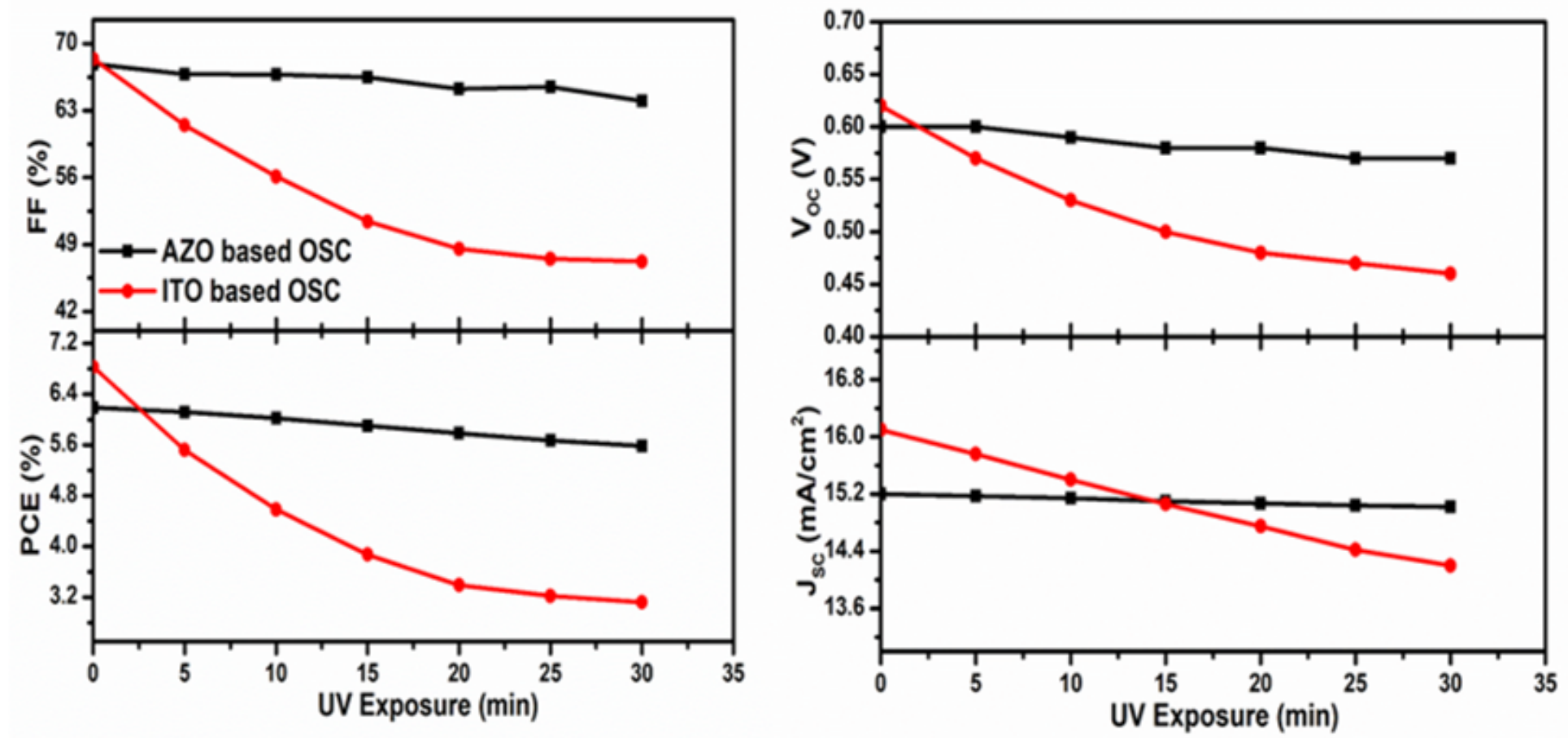

Figure 8

PCE, FF, $\mathrm{J}_{\mathrm{SC}}$ and $\mathrm{V}_{\mathrm{OC}}$ versus UV exposure time for AZO and ITO based inverted OSCs. 